\title{
BMJ Open Choosing Healthcare Options by Involving Canada's Elderly: a protocol for the CHOICE realist synthesis project on engaging older persons in healthcare decision-making
}

\author{
Paul Stolee, ${ }^{1,2}$ Jacobi Elliott, ${ }^{1}$ Heather McNeil, ${ }^{1}$ Veronique Boscart, ${ }^{1,2,3}$ \\ George A Heckman, ${ }^{1,2}$ Rebecca Hutchinson, ${ }^{4}$ Margaret Hedley, ${ }^{5}$ \\ Sholom Glouberman, ${ }^{6}$ Maria Judd ${ }^{7}$
}

To cite: Stolee P, Elliott J, McNeil $\mathrm{H}$, et al. Choosing Healthcare Options by Involving Canada's Elderly: a protocol for the CHOICE realist synthesis project on engaging older persons in healthcare decision-making. BMJ Open 2015;5:e008190. doi:10.1136/bmjopen-2015008190

- Prepublication history for this paper is available online. To view these files please visit the journal online (http://dx.doi.org/10.1136/ bmjopen-2015-008190)

Received 14 March 2015 Revised 2 October 2015 Accepted 16 October 2015

CrossMark

For numbered affiliations see end of article.

Correspondence to Dr Paul Stolee; stolee@uwaterloo.ca

\section{ABSTRACT}

Introduction: While patient and citizen engagement has been recognised as a crucial element in healthcare reform, limited attention has been paid to how best to engage seniors - the fastest growing segment of the population and the largest users of the healthcare system. To improve the healthcare services for this population, seniors and their families need to be engaged as active partners in healthcare decisionmaking, research and planning. This synthesis aims to understand the underlying context and mechanisms needed to achieve meaningful engagement of older adults in healthcare decision-making, research and planning.

Methods and analysis: The CHOICE Knowledge Synthesis Project: Choosing Healthcare Options by Involving Canada's Elderly aims to address this issue by synthesising current knowledge on patient, family, and caregiver engagement. A realist synthesis will support us to learn from other patient and citizen engagement initiatives, from previous research, and from seniors, families and caregivers themselves. The synthesis will guide development or adaptation of a framework, leading to the development of best practice guidelines and recommendations for engagement of older people and their families and caregivers in clinical decision-making, healthcare delivery, planning and research.

Ethics and dissemination: The components of this protocol involving consultation with patients or caregivers have received ethics clearance from the University of Waterloo, Office of Research Ethics (ORE\#19094). After completion of the project, we will amalgamate the information collected into a knowledge synthesis report which will include best practice guidelines and recommendations for patient, family and caregiver engagement in clinical and health system planning and research contexts.

Results: Will be further disseminated to citizens, clinicians, researchers and policymakers with the help of our partners.

Trial registration number CRD42015024749.

\section{INTRODUCTION}

It is widely recognised that healthcare systems have not been well designed to serve older persons with chronic diseases and other serious illnesses, despite seniors being the major users. ${ }^{12}$ To support a transformation of the healthcare system from an acute, episodic model of care to a primary care model that emphasises disease prevention and appropriate management of chronic illness, engagement of seniors and families is essential.

Multiple terms have been used to refer to patient and citizen engagement, including client engagement, community engagement, public involvement and other variations. For the purpose of this paper, we will be using the term 'patient, family and caregiver engagement'. We recognise there are many definitions; however, we have chosen the definition of patient engagement by Gallivan et $a l^{3}$ that state, "a relative term subjectively defined by individuals or groups/organizations that are planning to actively involve patients and their families in various health care advisory committees or care decision making". This definition recognises the importance of engaging families along with patients as key participants in healthcare research, planning and clinical decisionmaking. This is of particular significance for the engagement of seniors, for whom family members and other members of their informal networks are key partners in care. ${ }^{4}$ A prior ethnography by the first two authors (the InfoRehab project; https://uwaterloo. ca/geriatric-health-systems-research-group/ research/inforehab) explored patient, family and caregiver engagement with healthcare providers as older adults 
transitioned across the care continuum following a hip fracture. ${ }^{5-7}$ We found a consistent lack of meaningful engagement of patients and their families and caregivers in decision-making related to care.

We propose a synthesis of available knowledge on experiences of patient/family and caregiver engagement in the healthcare system, with a focus on seniors and older patients, and their family members and caregivers. By examining these experiences, including the processes, tools and methods, we aim to discern the underlying mechanisms by which meaningful engagement occurs. Our work will address two broad and interrelated categories of engagement: (1) engaging older patients and their families and caregivers in decisions around their own healthcare; and (2) engaging older persons, family members and caregivers in healthcare system planning and related research. This synthesis aims to answer the following research question: What are the contexts and underlying mechanisms needed to achieve the outcome of meaningful engagement of older adults in healthcare research, planning and decision-making? Specifically, we want to understand: (1) the contextual factors that influence meaningful engagement; (2) the outcome (levels of engagement) achieved through various engagement encounters; and (3) the mechanisms necessary to achieve meaningful engagement in healthcare decision-making, research and planning. From this synthesis, we aim to develop a framework for engagement efforts, and identify best practice guidelines to support engagement of older adults and their informal caregivers in healthcare decision-making, research and planning.

\section{STUDY PROTOCOL}

There is currently no consensus on the best approach to conduct a knowledge synthesis. ${ }^{8}$ Kastner et at propose a scoping review to identify the most appropriate knowledge synthesis method (the preliminary search for their scoping review included in their protocol paper describes 25 approaches). Of these approaches, we believe the realist synthesis approach, developed by Pawson $e t a l^{10}$ and Greenhalgh et $a l,{ }^{11}$ to be most appropriate for the purpose of this study. Realist syntheses provide the richness of information and explanation that can guide real-world decision-making. Realist syntheses address limitations of more traditional approaches to systematic reviews and meta-analyses which address effectiveness, often narrowly defined, but do not address why, for whom and in what circumstances and respects an intervention or policy does or does not work.

For our focus on engagement strategies for patient, family and caregiver in the healthcare delivery and research planning, a realist synthesis will allow for consideration of what engagement approaches may work for specific subgroups (eg, patients with cognitive impairment; caregivers living at a distance) in different healthcare settings (eg, primary healthcare, acute care, long-term care) and locations (eg, rural, urban). This type of review also allows us to consider different purposes of engagement, including patient engagement in decision-making around their healthcare, or in engagement in healthcare research.

Key to this approach is that strategies for patient, family and caregiver engagement are both the focus of our review and essential elements of our methodology. In our research group's efforts to engage seniors in healthcare research, we have developed the Seniors Helping as Research Partners (SHARP) network (www.uwaterloo.ca/ ghs/sharp). Since its inception in June 2013 we have recruited over 60 older adults to be involved in our research and planning efforts. This group developed from the partnerships and collaborations we have developed within the community, and the feedback received from these community members on their desire to be more involved in healthcare-related research. We will consult with interested members of this group in our synthesis (described below).

The basic phases of a realist review are similar to those followed in a conventional Cochrane review, but involve more substeps, and those steps may be overlapping and iterative rather than sequential. ${ }^{10}$ Realist synthesis involves identifying a theoretically based framework, which is then 'populated' with evidence that is used to enrich, adapt and/or refine the theory. The refined theory then becomes the basis for the best practice guidelines.

We note that the processes for this project of scope clarification, stakeholder involvement, systematic search and review processes, and development and dissemination of recommendations are consistent with accepted practice for development of best practice guidelines. ${ }^{12}$ Our knowledge synthesis will encompass a review of peer-reviewed and grey literature, conceptual/theoretical as well as empirical work; research conducted using qualitative, quantitative and mixed methods; as well as inviting expert opinion, including the opinions of seniors, older patients, and their families and caregivers. Knowledge generation then will consist of the adaption/ development of a framework for engagement, and knowledge translation is focused on the development of the best practice guidelines.

The first step in conducting a knowledge synthesis consists of establishing a review team including clinical and/or content experts (GAH, VB, MJ, PS, MH and $\mathrm{SG})$; determining participants experienced in synthesis methods, systematic reviews and knowledge syntheses (PS, GAH, VB and RH); selecting researchers who have conducted primary research in the area (SG,VB, JE, HM and PS); appointing/selecting a librarian/information scientist $(\mathrm{RH})$, knowledge users and consumer representatives (SG and $\mathrm{MH}$ ). Patients Canada (SG) and the Canadian Foundation for Healthcare Improvement (CFHI, MJ) will provide opportunities for dissemination and implementation of findings through their respective networks. The CFHI (http://www.cfhi-cass.ca/ 
WhatWeDo/Collaborations/PatientEngagement.aspx) actively promotes patient engagement in healthcare throughout Canada. Patients Canada is leading efforts to increase understanding of patient experience (patientsassociation.ca/stories). ${ }^{13}$

The five major phases of a realist synthesis are outlined, along with the elements of our proposed research project.

\section{Phase 1: clarifying scope}

This step involves in-depth discussion with stakeholders to refine the review question and purposes of the review, and to find and articulate relevant theories. Following the protocol for a realist review, the candidate theories (or 'initial rough theories, ${ }^{14}$ ) will guide the understanding of the context-mechanism-outcome relationship for engaging older adults. Initial theories, such as the spectrum of engagement, ${ }^{15}$ were identified during preliminary searches. A variety of methods will be used to derive the list of candidate theories including brain storming within the review team, performing a snowball search of the grey literature on Google and consulting with experts in this area (both academics and older adults). All of the identified frameworks will be reviewed at a workshop with older adults and caregivers.

Grey literature sources will be selected based on our understanding of where current work in the field is taking place, as well as through preliminary internet searches. Our focus is on Canadian information at the provincial and national levels, but we will also pay close attention to research identified through database and hand searching from countries with a similar healthcare system. We will use this information to identify other government websites and search them for relevant reports. The UK has produced a significant amount of research on public engagement in healthcare decisions, so UK-based websites will be a deliberate focus of our search. A search will be conducted to find guidelines, government reports and conference proceedings using these websites as a starting point: The UK National Institute for Health and Care Excellence; the UK Social Care Institute for Excellence; UK Department of
Health, Public Health Agency of Canada; CFHI; Canadian Institutes of Health Research; National Collaborating Centres for Public Health; and Patient Voices Network. We will also search public health association websites and government health websites for each province and territory. We will search the websites for documents using relevant keywords (table 1), and then search for the keywords within the found documents to identify relevant information.

Interviews will be conducted with key research leaders who have expertise in patient, family and community engagement in Canada and abroad to help us review theories, frameworks and strategies that could help to guide our search strategy. As well, during this phase of the project, we will consult with older adults and caregivers from our SHARP network. We will look to these participants for input on engagement and what it means to them as the consumer. A 1-day workshop will be conducted with the study team, and members of Patients Canada to refine review questions and purposes. The workshop will allow participant input and discussions on existing relevant theories that could be used to guide the synthesis.

\section{Phase 2: search for evidence}

An extensive, purposive search of peer-reviewed literature will be conducted. We recognise that as information is retrieved, this may identify areas where additional focused searching may be required.

\section{Search methodology}

The knowledge synthesis will focus on strategies for engaging older adults and their families and caregivers in healthcare decision-making. Healthcare decisionmaking pertains to the research, planning and policies within the healthcare system, as well as clinical decisionmaking at the patient level. The information we retrieve will be divided into broader healthcare system engagement and narrower patient-level decision-making. The search methodology was informed by the quality standards and guidelines for realist syntheses put forward by Wong et al. ${ }^{1416}$

Table 1 Search terms

\begin{tabular}{|c|c|}
\hline Concept $^{*}$ & Relevant key wordst \\
\hline $\begin{array}{l}\text { Healthcare } \\
\text { decision-making }\end{array}$ & $\begin{array}{l}\text { healthcare, health care, health services, health system, health care services, health care system } \\
\text { Decision making, research, planning, policy, reform, delivery, development, organizational change } \\
\text { delivery of health care, health care reform, health care evaluation mechanisms, health care delivery, } \\
\text { health care planning }\end{array}$ \\
\hline Public engagement & $\begin{array}{l}\text { person-centred care, patient-centred care, patient and family centred care community-based } \\
\text { participatory research } \\
\text { public, citizen, consumer, user, community, patient } \\
\text { engagement, involvement, participation, empower, representation, collaboration }\end{array}$ \\
\hline
\end{tabular}


Preliminary searches have indicated that 'older adults' or related phrasing is not often included in titles or abstracts. Our search will encompass the broader scope of all public engagement, and the age demographic will be considered in analysis stage. Using the snowballing method, we will conduct a hand search to identify possible relevant citations from the reference lists of pertinent papers and studies, paying close attention to the reference lists of review articles. An initial list of search terms is provided in table 1 .

Search results will be exported to RefWorks, a type of reference management software, and duplicate results deleted. In the first screening, multiple reviewers will read through the abstracts and apply the inclusion and exclusion criteria to decide if the full article should be retrieved. If there is uncertainty, this will be resolved in discussion with another reviewer, and if necessary, with the principal investigator. If there is not enough information to make an informed decision, the article will be retrieved. In the second screening, the inclusion/exclusion criteria will be applied to the full text-articles. To pilot test consistent application of eligibility criteria, inter-reviewer agreement on article retention will be assessed. A sample of articles will first be reviewed (full text) independently by two reviewers and results compared using a $\kappa$ statistic. ${ }^{9}$ This process will be repeated and discrepancies discussed among reviewers until a $\kappa$ statistic considered as 'good' using Altman's criteria $(>0.60)$ is achieved. ${ }^{17}$

\section{Peer-reviewed journal databases}

The date limits of the literature searched will range from the earliest coverage of individual databases to the date of the final search. The review will include both English and French language content.

A systematic search will be conducted in the following licensed databases: MEDLINE (1946-present) and EMBASE (1974-present) will be searched through the Ovid interface, so subject headings (MeSH and EMTREE) and proximity operators can be utilised. Other databases include CINAHL (1981-present), Sociological Abstracts (1952-present), Scopus (most sources covered from 1996 to present, with some indexed back to mid-1900s), which contains journal articles as well as a large collection of conference proceedings, and the Cochrane Database of Systematic Reviews (1994-present).

\section{Inclusion and exclusion criteria}

Papers will be included that report description, assessment or evaluation of strategies for engagement of adults (18+ patients or citizens, families or caregivers). Any and all papers that discuss outcomes that could discern an actual experience or process of engagement (ie, not papers that describe the importance of engagement) will be included. To be as comprehensive as possible, we will initially review papers relevant to all adult age groups because preliminary searches show a limited body of evidence specific to older adults. There is the potential for work done in broader age groups to have relevance to older adults. Additional steps in the review will allow the researchers to consult with older adults to assess the relevance of information for the older adult population. Papers containing strategies relevant to older adults (age 65+) will be highlighted in our abstraction. Papers focusing strictly on engagement of children (age $<18$ ) will be excluded. The inclusion/ exclusion criteria may be modified by our scope clarification phase. As well, initial searches may identify areas where additional focused searches are warranted.

\section{Phase 3: appraise primary studies and extract data}

In realist syntheses, data abstraction is an ongoing, iterative process that is dependent on information gathered. Data abstraction, including an assessment of relevance and rigour, will be conducted following guidelines outlined by Pawson et $a l^{10}$ and by Wong et al. ${ }^{14}{ }^{16}$ Bespoke abstraction forms will be developed based on the scope clarification phase and relevant theories of engagement. The data abstraction forms will also gather information about context-mechanism-outcome configurations, contextual information, study characteristics, patient characteristics and intervention characteristics. Citations will be divided into two areas of focus (context) - engagement in clinical decision-making, and engagement in research or health policy-making. This will allow the syntheses to be specific enough to understand the underlying mechanisms of the implementations of engagement for each context. We will assess for relevance, driven by how each study contributes to theory testing, by asking questions suggested by Kastner $e t a l,{ }^{8}$ such as: Does the research address the theory under investigation? In what context does the engagement occur? Does the engagement involve older patients, family or caregivers? Consistent with the recommendation of Kastner et al, ${ }^{8}$ rigour will be considered a 'mediating tool rather than a testing method for article selection'. Rigour is used in a realist synthesis to apply judgement to the articles being reviewed to assess their quality-Does the research support the conclusions drawn from it? ${ }^{810} 1416$

\section{Phase 4: analyse and synthesise evidence}

The search results will be used to 'populate' the candidate theory or theories with available evidence and experience. To do this, we will consider how the evidence illuminates the workings of the initial theories, identify aspects of the theories that may not hold up after consideration of evidence and develop a coherent understanding. The data analysis and synthesis will follow a process similar to that described by Wong et al. ${ }^{18}$ We will use Nvivo V.10 software to examine the extracted data and identify and code themes, ideas and concepts that emerge through our reading. We will examine the information related to the context (specific populations, geographies, organisations involved in research, planning or clinical decision-making), potential mechanisms 
and outcomes (meaningful engagement of older adults in healthcare). We will also look for patterns (demi-regularities) that might help us understand how to achieve meaningful engagement. This qualitative examination will involve active comparison across programmes and contexts. The data synthesis process will involve team discussions and reflections, where we will review and consider the value of the codes/themes and how they contribute to the meaning of the themes related to the candidate theories. We will look for data that either support or counter the original theories. The researchers will synthesise the findings into summary reports for review in the final phase.

\section{Phase 5: disseminate, implement and evaluate}

The final phase of the realist synthesis will engage stakeholders to review findings, and to gain their insights into how the information can be used to achieve outcomes of meaningful engagement. Potential examples might include:

1. Engagement in decision-making around treatment options for a particular disease

- Context-patient presenting for first time in physician's office with congestive heart failure;

- Mechanism-respectful communication;

- Outcome-a relationship of trust between patient and physician.

2. A collaborative research project involving older adult participants

- Context: collaborative research project of academic researchers and older citizens;

- Mechanism: mutual valuing of knowledge;

- Outcome: feeling of belonging on research team.

This stage will include a repeat consultation session with SHARP members and a follow-up 1-day workshop with the study team and Patients Canada members to review and interpret study findings and consider their potential application in various contexts (eg, urban vs rural, hospital vs community, clinical vs policy). After completion of these activities, we will amalgamate the information collected into a knowledge synthesis report which will include the refined theory, and guidelines and recommendations that may emerge from theory to support patient/family and caregiver engagement in clinical and health system planning/research. Results will be further disseminated to citizens, clinicians, researchers and policymakers with the help of our partners. Our review has been registered with PROSPERO, CRD42015024749.

\section{Anticipated challenges}

A potential challenge to this knowledge synthesis may be that the second phase will present with an unmanageable number of articles generated by the search. If this is the case, we may consult with our partners and stakeholders to refine and focus our search strategy. If the number of articles identified for review is still impractical, it may be necessary to use a method of theoretical saturation for a realist review. ${ }^{19}$
Another potential challenge may be related to engaging older adults and caregivers throughout phases 1 and 4 of our review. Our team is committed to engaging older adults in this project. We recognise that when working with older adults, there may be mobility limitations that present a challenge when arranging engagement/consultation sessions. To overcome these, we will plan to host these at convenient, accessible locations during a time that is convenient to the older adults. Our team has experience with consultations of over 600 community members. Members of the SHARP network have expressed eagerness to participate in our research. Our partner Patients Canada has extensive experience assembling panels of patients and family members for consultation.

\section{DISCUSSION}

As noted earlier, there is currently no consensus on an agreed methodology for knowledge syntheses. ${ }^{9}$ With growing interest in knowledge syntheses including realist syntheses, the sharing of study protocols such as this one is important. We see the direct engagement of patients and families in this synthesis effort as a key strength of our methodology.

This review will provide an understanding of key principles that promote the engagement of older adults and their caregivers as equal partners in healthcare research, planning and decision-making. The synthesis will generate a framework on which we build: (1) strategies and best practice guidelines for engagement where these can be supported by available knowledge; and (2) future research where additional knowledge or evidence is needed. We believe that it is essential to consider engagement broadly-patients and citizens need to be able to engage in decision-making around their own care, and they also need to engage in developing a healthcare system in which this type of involvement is accommodated and respected. This project recognises patient, family and caregiver engagement as a key health system improvement strategy. Research has found evidence of impacts of engagement on healthcare services $^{20}$ and patient outcomes, ${ }^{21}{ }^{22}$ as well as benefits of engagement for participants. ${ }^{23}$ Nonetheless, there is a recognition of a need for a greater understanding of the theoretical underpinnings of engagement ${ }^{20}$ and of the best methods by which engagement can be achieved. ${ }^{24}$ This review will provide an understanding of key principles that promote the engagement of older adults and their caregivers as equal partners in healthcare research, planning and decision-making. A wide range of stakeholders will benefit from this study, including healthcare providers, investigators involved in healthcare research and planning (in academic, government or other organisations), decision-makers, and older adults and their caregivers. We plan to use the results of this synthesis to guide our own research efforts, including our engagement with the SHARP group. 


\section{ETHICS AND DISSEMINATION}

The findings from this knowledge synthesis will yield best practice guidelines for engagement leading to more productive partnerships and collaborations among patients/families and caregivers, healthcare providers, health system decision-makers and planners, and researchers. Results will be disseminated through a number of different avenues including peer-reviewed publications in academic journals, presentations at national and international conferences, lay summaries and forums for community stakeholders.

\section{Author affiliations}

${ }^{1}$ School of Public Health and Health Systems, University of Waterloo, Waterloo, Ontario, Canada

${ }^{2}$ Schlegel-UW Research Institute for Aging, Kitchener, Ontario, Canada

${ }^{3}$ School of Health and Life Sciences and Community Services, Conestoga College, Kitchener, Ontario, Canada

${ }^{4}$ Library Services, University of Waterloo, Waterloo, Ontario, Canada

${ }^{5}$ Guelph-Wellington Seniors Association, Guelph, Ontario, Canada

${ }^{6}$ Patients Canada, Toronto, Ontario, Canada

${ }^{7}$ Canadian Foundation for Healthcare Improvement, Ottawa, Ontario, Canada

Acknowledgements All the authors acknowledge the support provided by Patients Canada and the Canadian Foundation for Healthcare Improvement.

Contributors JE and $\mathrm{HM}$ are $\mathrm{PhD}$ students who assisted in designing the project. RH provided the initial search strategy for the protocol submission. $\mathrm{MH}, \mathrm{SG}$ and MJ are knowledge users who have provided expertise towards the design of this project and will assist where necessary throughout the project.

Funding This project is funded by the Technology Evaluation in the Elderly Network (TVN, grant \# KS2013-08), which is funded by the Government of Canada's Networks of Centres of Excellence (NCE) Program.

Competing interests None declared.

Ethics approval University of Waterloo, Office of Research Ethics (ORE\# 19094).

Provenance and peer review Not commissioned; externally peer reviewed.

Open Access This is an Open Access article distributed in accordance with the Creative Commons Attribution Non Commercial (CC BY-NC 4.0) license, which permits others to distribute, remix, adapt, build upon this work noncommercially, and license their derivative works on different terms, provided the original work is properly cited and the use is non-commercial. See: http:// creativecommons.org/licenses/by-nc/4.0/

\section{REFERENCES}

1. Brand C, Scott I, Greenberg P, et al. Chronic disease management: time for consultant physicians to take more leadership in system redesign. Intern Med J 2007;37:653-9.

2. Heckman GA. Integrated care for the frail elderly. Healthc Pap 2011;11:62-8.

3. Gallivan J, Kovacs Burns K, Bellows M, et al. The many faces of patient engagement. J Particip Med 2012;4:e32.
4. Levine C, Halper D, Peist A, et al. Bridging troubled waters: family caregivers, transitions, and long-term care. Health Aff 2010;29:116-24.

5. Elliott J, Forbes D, Chesworth B, et al. Information sharing with rura family caregivers during care transitions of hip fracture patients. Int $J$ Integr Care 2014;14:e018.

6. Toscan J, Manderson B, Santi SM, et al. "Just another fish in the pond": the transitional care experience of a hip fracture patient. Int $J$ Integr Care 2012;13:e023.

7. Hicks E, Sims-Gould J, Byrne K, et al. "She was a little bit unrealistic": choice in healthcare decision making for older people. $J$ Aging Stud 2012;26:140-8.

8. Kastner M, Estey E, Perrier L. Understanding the relationship between the perceived characteristics of clinical practice guidelines and their uptake: protocol for a realist review. Implement Sci 2011;6:69.

9. Kastner M, Tricco A, Soobiah C, et al. What is the most appropriate knowledge synthesis method to conduct a review? Protocol for a scoping review. BMC Med Res Methodol 2012;12:114.

10. Pawson R, Greenhalgh T, Harvey G, et al. Realist review-a new method of systematic review designed for complex policy interventions. J Health Serv Res Policy 2005;10(Suppl):21-34.

11. Greenhalgh T, Wong G, Westhorp G, et al. Protocol-realist and meta-narrative evidence synthesis: evolving standards (RAMESES). BMC Med Res Methodol 2011;11:115.

12. Brouwers M, Kho ME, Browman GP, et al. Next Steps Consortium AGREE II: advancing guideline development, reporting and evaluation in healthcare. CMAJ 2010;182:E839-842.

13. Glouberman S. My operation: a health care insider becomes a patient. Toronto, ON: Health and Everything Publications, 2011.

14. Wong G, Westhorp G, Pawson R, et al. Realist synthesis: RAMESES training materials. http://www.ramesesproject.org/media/ Realist_reviews_training_materials.pdf

15. International Association for Public Participation (IAP2). Spectrum of participation. 2007. http://c.ymcdn.com/sites/www.iap2.org/resource/ resmgr/imported/IAP2\%20Spectrum vertical.pdf (accessed 20 Sept 2013).

16. Wong G, Greenhalgh T, Westhorp G, et al. Development of methodological guidance, publication standards and training materials for realist and meta-narrative reviews: the RAMESES (Realist And Meta-narrative Evidence Syntheses-Evolving Standards) project. Health Serv Deliv Res 2014;2:1-252.

17. Altman DG. Practical statistics for medical research. London: Chapman and Hall, 1991.

18. Wong G, Greenhalgh T, Pawson R. Internet-based medical education: a realist review of what works, for whom and in what circumstances. BMJ Med Educ 2010;10:12.

19. Pawson R, Greenhalgh T, Harvey G, et al. Realist synthesis: an introduction. ESRC Research Methods Programme. Manchester: University of Manchester, 2004.

20. Mockford C, Staniszewska S, Griffiths F, et al. The impact of patient and public involvement on UK NHS health care: a systematic review. Int J Qual Health Care 2012;24:28-38.

21. Durand $M$, Carpenter L, Dolan $\mathrm{H}$, et al. Do interventions designed to support shared decision-making reduce health inequalities? A systematic review and meta-analysis. PLOS ONE 2014;9: e94670.

22. Shah R, Franks $P$, Jerant $A$, et al. The effect of targeted and tailored patient depression engagement interventions on patient-physician discussion of suicidal thoughts: a randomized control trial. J Gen Intern Med 2014;29:1148-54.

23. Brett J, Staniszewska S, Mockford C, et al. A systematic review of the impact of patient and public involvement on service users, researchers and communities. Patient 2014;7:387-95.

24. Domecq J, Prutsky G, Elraiyah T, et al. Patient engagement in research: a systematic review. BMC Health Serv Res 2014;14:89. 